\title{
The Effects of Atmospheric Plasma on Microbes, Mold and Yeast in Powdered Protein
}

\author{
Heidi Lightfoot
}

Clinical Research Physician, Basingstoke and North Hampshire Hospital, England Email: heidi.lightfoot@gmail.com 


\begin{abstract}
:
Foodborne illnesses can manifest as irritation and infections of the gastrointestinal tract, caused more specifically by harmful bacteria, parasites, viruses or chemicals found in food items. The reduction of microbes, mold and yeast could therefore greatly increase food safety and lower the incidence of foodborne illnesses worldwide. Plasma-surface modification (PSM) has been used as a method to alter surface structure of materials, changing both their external and internal properties and potentially reducing the amount of mold, microbes or yeast in food products.

The purpose of this study was to investigate the effect of PSM on the amount of mold and yeast, as well as microbes found on powdered protein upon treatment. Given the wide spectrum of applications of powdered food, minimizing undesired microbes, mold, and yeast that may be found in the product could be of significant consequence. Here we report a nearly total reduction in the mold and yeast count following one and three ioShield treatments. The microbe count had a more incremental response; one ioShield treatment partially reduced the count and three treatments lowered the amounts of microbes almost entirely. This indicates that treatment with atmospheric plasma is an effective way to reduce mold and yeast as well as microbial contaminants, and as such is an effective means to minimize foodborne illnesses and increase food safety.
\end{abstract}




\section{Figures:}

Figure A

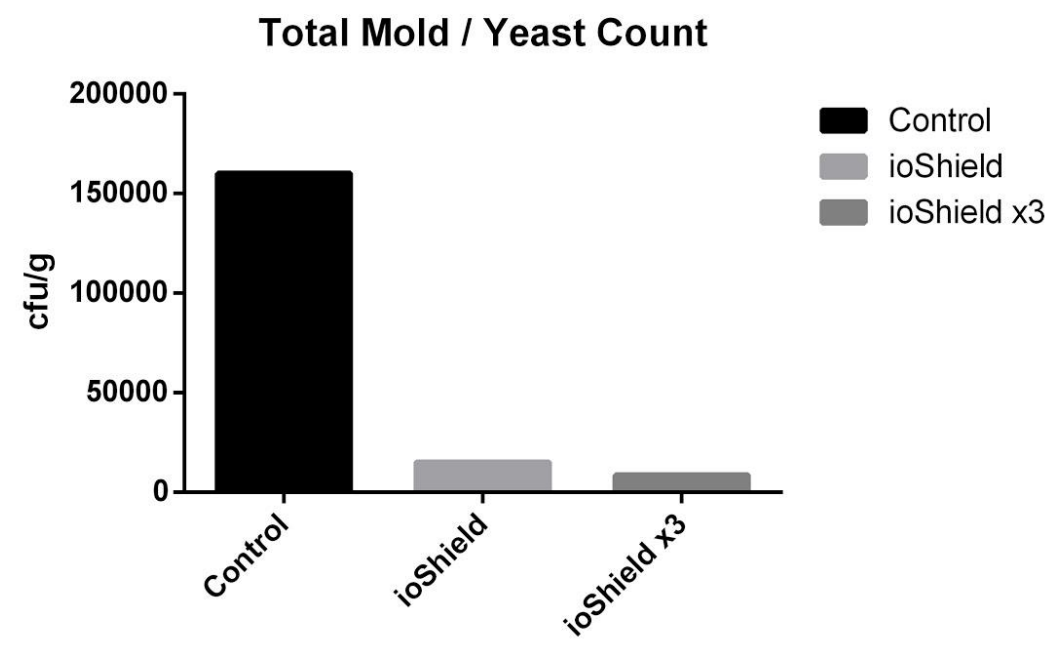

Figure B

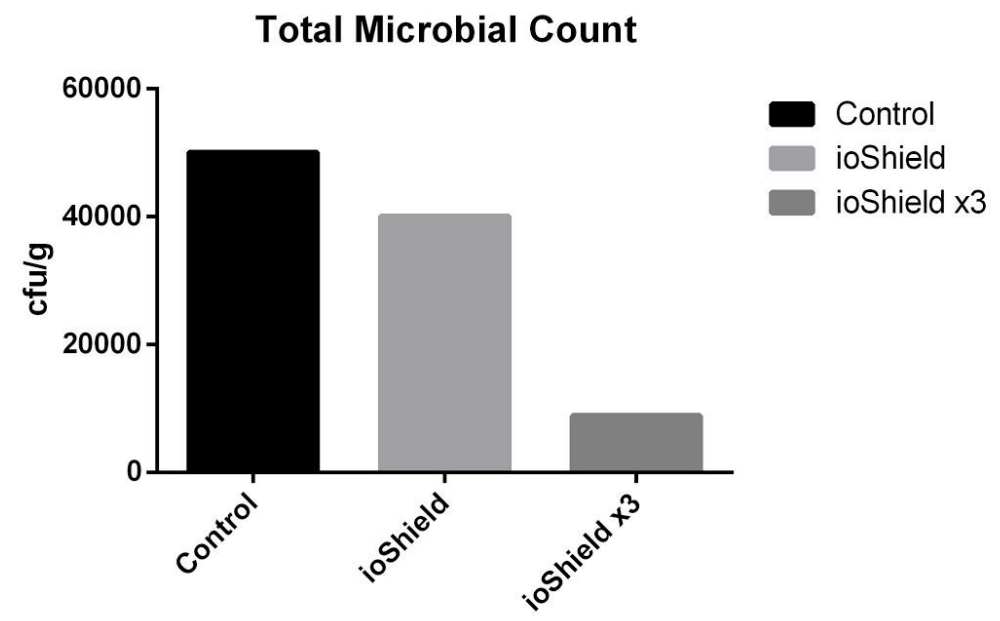

Figure Legend:

Figure A: Comparison of the amount of mold and yeast on untreated and plasmatreated protein. A significant decrease in mold and yeast is achieved after one and three ioShield treatments.

Figure B: Comparison of microbe amounts on untreated and plasma-treated protein. A decrease in microbe count is achieved after one ioShield treatment and a significant reduction is observed after three treatment rounds.

\section{Introduction:}

Food safety represents a major ongoing public health concern worldwide, in both developed and developing countries; contaminated food is a heavy healthcare burden 
both socially as well as economically. Food, and in particular fresh food such as produce or meat, is prone to spoilage due to contaminants such as mold, yeast and bacteria, all of which reduce shelf life. Foodborne illnesses affect millions of Americans each year'; these illnesses are caused primarily by the presence of bacteria and viruses in ingested food.2 With the recent increased awareness of food safety concerns, technologies that effectively reduce potentially harmful mold, yeast and bacteria without altering taste or nutrition are in high demand. The use of plasma as an effective treatment to both prolong shelf-life and reduce pathogens has recently caught the attention of both the research community as well as commercial sectors.

Numerous other studies have investigated the potential positive impact of plasma treatment on food safety. Investigation of the use of plasma as a food processing aid has included applications such as decontamination ${ }^{3}$, inactivation of enzymes ${ }^{4}$, toxin elimination ${ }^{5}$, package optimization ${ }^{6}$ and water purification' ${ }^{7}$. Cold plasma, a nonthermal and sustainable technology, has also recently been highlighted as an effective means to eliminate microbes on foods such as meat, poultry, fruits and vegetables. This technology has been investigated as an effective method in food preservation ${ }^{8}$ as well as a mediator of foodborne pathogens. ${ }^{9}$ The effects of argon, water vapor and air plasma on several bacterial species in brown rice cereal were also investigated, whereby air plasma was found to be the most efficacious in extending shelf-life. ${ }^{10}$ Another recent study has found that non-thermal atmospheric plasma was an effective inactivator of bacterial cultures found on eggs." Furthermore, a surface discharge cold plasma device has been shown to be an efficient inactivator of major foodborne pathogens such as Escherichia coli and Listeria monocytogenes. ${ }^{12}$

Plasma is the highly energized fourth state of matter. It is highly conductive and consists primarily of highly ionized gases. Plasma-surface modification (PSM) is a process of altering chemical and biophysical properties of materials by means of using plasma. During treatment with plasma, the ionized gases come into contact with the surface of a material, causing structural alterations that in turn cause intrinsic and/or extrinsic characteristics of a given material to change ${ }^{13}$. If the ionization occurs at room temperature, the plasma treatment is said to be atmospheric. ${ }^{14}$ So far, PSM has been investigated as a method to modify functional properties of organic and inorganic materials, but its use in powdered food processing has not yet been researched.

\section{Objective:}

The goal of this study is to investigate whether atmospheric plasma is an effective way to increase food safety by means of reducing mold, yeast and bacteria counts in powdered protein.

\section{Results:}

Samples were exposed to plasma treatment via proprietary PSM technology (Ingredient Optimized $\mathrm{r}$, Plasma Nutrition, USA) and mold, yeast and microbial counts were assessed. Source material was obtained and separated into treated and non-treated categories. Mold, yeast and microbial counts were compared relative to control following one and three rounds of treatment with plasma. 
Total aerobic microbial count and total yeast/mold count were tested using the current USP, microbial limit tests (61) by a third party and independent lab.

Mold and yeast counts were reduced significantly following one round of PSM treatment and even further reduced after three rounds (Figure A). Microbial counts appeared more resistant, with a modest count reduction after one treatment and a significant decrease after three rounds of PSA treatments (Figure B).

\section{Discussion:}

There are various applications of plasma as a food processing aid including food packaging, decontamination, and functionalization. ${ }^{15}$ Here we examine the ability of PSM to reduce undesired agents such as mold, yeast and bacteria directly on food. Our results highlight the reduction by PSM on all three, indicating that it might be an efficacious method to minimize foodborne pathogens. This is of particular interest in the food and healthcare industries that are continually seeking effective means by which to increase food safety, as well as lower contamination due to mold and bacteria.

\section{Conclusions:}

Mold, yeast, and bacteria are some of the most frequently encountered food contaminants and can contribute to the incidence of foodborne illnesses. The amount of each of these contaminants was significantly decreased following treatment with atmospheric plasma. An enhanced reduction in mold and yeast was achieved following multiple rounds of treatment; while a modest decrease was obtained after one round, nearly all of the bacteria was eliminated after three treatment rounds. These findings are strong implications for the use of atmospheric plasma as a supplemental method to reduce contaminants found in food and thereby possibly to lower the frequency of food borne illnesses.

\section{Methods:}

Total aerobic microbial count and total yeast/mold count were tested using the current USP, microbial limit tests (61) by a third party and independent lab.

\section{Limitations:}

This study investigated the application of atmospheric plasma as a way to reduce the amounts of mold, yeast, and bacteria on one source of dry powdered protein. Given that this is just one kind of an otherwise commercially available source material, the use of other similar products may yield results that are somewhat different from those we report here. These variations can be addressed with examining the effects of atmospheric plasma on mold, yeast and bacteria found on a range of dry powder protein products.

\section{Funding Statement:}

This work was financially supported by Plasma Nutrition. 


\section{Ethics Statement:}

No fraudulence is committed in performing these experiments or during processing of the data.

\section{Sources:}

1. Scallan, E., Griffin, P.M., Angulo, F.J., Tauxe, R.V. and Hoekstra, R.M. (2011). Foodborne illness acquired in the United States-unspecified agents. Emerging infectious diseases, 17(1), p.16.

2. Boore, A., Herman, K.M., Perez, A.S., Chen, C.C., Cole, D.J., Mahon, B.E., Griffin, P.M., Williams, I.T. and Hall, A.J. (2010). Surveillance for foodborne disease outbreaks-United States, 2007. Morbidity and Mortality Weekly Report, 59(31), pp.973-979.

3. Misra, N.N., Tiwari B.K., Raghavarao K.S.M.S. and Cullen P.J. (2011). Nonthermal plasma inactivation of food-borne pathogens. Food Eng Rev (3), pp. 159170. DOI: 10.1007/s12393-011-9041-9

4. Misra, N.N., Pankaj, S.K. and Segat, A. (2016). Ishikawa, K. Cold plasma interactions with enzymes in foods and model systems. Trends Food Sci. Technol., 55, pp. 39-47. DOI: 10.1016/j.tifs.2016.07.001

5. Misra, N.N. (2015). The contribution of non-thermal and advanced oxidation technologies towards dissipation of pesticide residues. Trends Food Sci. Technol., 45, pp. 229-244. DOI: 10.1016/j.tifs.2015.06.005

6. Pankaj, S.K., Bueno-Ferrer, C., Misra, N.N.; Milosavljevic', V., O'Donnell, C.P., Bourke, P., Keener, K.M. and Cullen, P.J. (2014). Applications of cold plasma technology in food packaging. Trends Food Sci. Technol., 35, pp. 5-17. DOI: 10.1016/j.tifs.2013.10.009

7. Sarangapani, C., Misra, N.N., Milosavljevic, V., Bourke, P., O’Regan, F. and Cullen, P.J. (2016). Pesticide degradation in water using atmospheric air cold plasma. J. Water Process Eng., 9, pp. 225-232. DOI: 10.1016/j.jwpe.2016.01.003

8. Niemira, B. A. (2012). Cold plasma decontamination of foods. Annual review of food science and technology, 3, pp. 125-142. DOI: 10.1146/annurev-food-022811101132

9. Misra, N. N., Schlüter, O., \& Cullen, P. J. (Eds.). (2016). Cold plasma in food and agriculture: Fundamentals and applications. Academic Press.

10. Suhem, K., Matan, N., and Nisoa, M. (2013). In vitro and in vivo antifungal activities of various gas species under plasma jet treatment against brown rice cereal spoilage molds. International Food Research Journal, 20(2), pp. 947-951.

11. Dasan, B.G., Yildirim, T. and Boyaci, I.H. (2018). Surface decontamination of eggshells by using non-thermal atmospheric plasma. International journal of food microbiology, 266, pp.267-273. DOI: 10.1016/j.ijfoodmicro.2017.12.021

12. Timmons, C., Pai, K., Jacob, J., Zhang, G. and Ma, L.M. (2018). Inactivation of Salmonella enterica, Shiga toxin-producing Escherichia coli, and Listeria monocytogenes by a novel surface discharge cold plasma design. Food Control, 84, pp.455-462. DOI: 10.1016/j.foodcont.2017.09.007 
13. Shenton, M.J. and Stevens, G.C. (2001). Surface modification of polymer surfaces: atmospheric plasma versus vacuum plasma treatments. Journal of Physics D: Applied Physics, 34(18), p.2761. DOI: 10.1088/0022-3727/34/18/308

14. Chu, P.K., Chen, J.Y., Wang, L.P. and Huang, N. (2002). Plasma-surface modification of biomaterials. Materials Science and Engineering: R: Reports, 36(5), pp.143-206. DOI: 10.1016/S0927-796X(02)00004-9

15. Cullen, P. J., Lalor, J., Scally, L., Boehm, D., Milosavljević, V., Bourke, P., and Keener, K. (2018). Translation of plasma technology from the lab to the food industry. Plasma Processes and Polymers, 15(2), 1700085. DOI: $\underline{10.1002 / p p a p .201700085}$ 